\title{
Gallbladder wall thickening in infectious mononucleosis: an ominous sign
}

\author{
Neil O’Donovan, Edward Fitzgerald
}

\begin{abstract}
Summary
Gallbladder wall thickening $(3 \mathrm{~mm}$ or greater) is a nonspecific finding with many causes. We describe two cases caused by infectious mononucleosis. Other causes of gallbladder wall thickening are described and the literature is reviewed. We suggest that the finding of gallbladder wall thickening in a patient with infectious mononucleosis implies that the patient is very ill, and its observation should lead to close patient monitoring.
\end{abstract}

Keywords: gallbladder wall thickening, infectious mononucleosis, Epstein-Barr virus

Infectious mononucleosis is a viral infection caused by the Epstein-Barr virus which excites a reticuloendothelial reaction in the host. Though generally a relatively benign condition, in a small percentage of patients it can cause severe systemic illness, as in the two cases we have reported. The clinical spectrum of infectious mononucleosis includes fatigue, anorexia, lymphadenopathy and it may clinically mimic viral hepatitis. It usually occurs in young adults but when it occurs in older patients it can pose diagnostic problems. In these patients the lymphadenopathy is less pronounced. Our two cases encompass both age groups and you will see the presentations were somewhat different. In both cases however, marked gallbladder wall thickening was associated with severe systemic illness.

\section{Case reports}

Case 1

A 20-year-old woman was admitted with a fiveday history of anorexia, pyrexia, nausea and accompanying malaise. She was noted to be jaundiced on the day of admission. Liver function tests were abnormal with an elevated bilirubin, alkaline phosphatase and aspartate transaminase. Hepatitis studies were normal. Monospot test was positive, as was EpsteinBarr virus serology, which was IgM positive, indicating acute infection. Leptospirosis titre was negative.

An abdominal ultrasound scan (figure) showed marked thickening of the gallbladder wall. The remainder of the study was normal. The patient's symptoms gradually regressed over the next five weeks and her liver function tests returned to normal. A repeat ultrasound examination showed a normal gallbladder wall.
Case 2

A 46-year-old man was admitted with a sixweek history of intermittent sore throat, flu-like symptoms, fever and nausea. Examination revealed cervical and axillary lymphadenopathy as well as mild hepatomegaly. The patient was found to have a pansystolic murmur and crackles in both lung bases. Liver enzymes were markedly elevated and the serum albumin level was low. A significant leucocytosis was also noted and atypical lymphocytes were seen on the blood film. The Monospot test was positive. An abdominal ultrasound was performed which demonstrated a thickened oedematous gallbladder wall. No gallstones were visualized. The spleen was of normal size.

The patient developed acute pulmonary oedema which necessitated his transferral to the intensive care unit. During his stay in hospital he underwent two echocardiograms and a cardiac catheterization. Epstein-Barr virus serology was IgM positive. Blood cultures were negative. The patient was discharged following 18 days of hospitalization. The final diagnosis was acute Epstein-Barr virus infection with associated myocarditis.

\section{Discussion}

The fasting gallbladder wall in normal volunteers is 1 to $2 \mathrm{~mm}$ thick, the median value being $1.5 \mathrm{~mm}$. In no case in this series did gallbladder wall thickness exceed $3 \mathrm{~mm}$ after a fatty meal. ${ }^{1}$ Gallbladder wall thickening $(3 \mathrm{~mm}$ or greater) is a nonspecific finding with many causes (box 1). ${ }^{2-6}$ It may arise due to obstruction of lymphatic drainage' resulting in increased interstitial fluid and thickening of tissue. $^{2}$ In chronic liver disease and portal

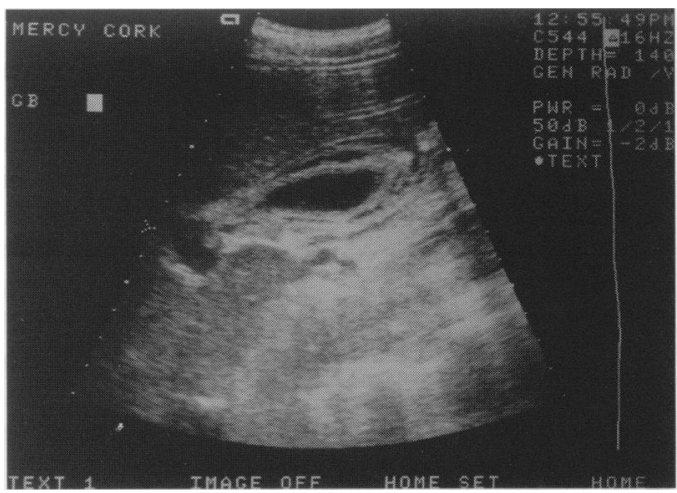

Figure This demonstrates marked gallbladder wall thickening and oedema with some pericholecystic fluid 


\section{Causes of increased thickness of the} gallbladder wall

- intrinsic gallbladder disease

- acute or chronic cholecystitis

- contracted gallbladder after eating

- acute hepatitis

- ascites

- hypoalbuminaemia

- congestive cardiac failure

- portal hypertension

- chronic liver disease

- multiple myeloma and acute myeloid leukaemia

- pyelonephritis

- infectious mononucleosis

\section{Box 1}

hypertension, hypoalbuminaemia is the dominant factor causing gallbladder wall thickening. This may have an anatomical basis, since the venous drainage of the gallbladder is via the right portal vein. ${ }^{1}$ Indeed demonstrations of gallbladder wall thickening in chronic liver disease should suggest the presence of portal hypertension. ${ }^{5}$

In both our cases, the gallbladder wall was diffusely thickened, measuring up to $8 \mathrm{~mm}$. This marked thickening was similar to that found in the other reported cases. ${ }^{7,8}$ The thickening of the gallbladder wall in infectious

1 Ralls PW, Quinn MF, Juttner HU, et al. Gallbladder wall thickening: patients without intrinsic gallbladder disease. AfR 1981; 137: 65-8.

2 Carroll B. Gallbladder wall thickening secondary to focal lymphatic obstruction. F Ultrasound Med 1983; 2: 89-91.

3 Fitzgerald EJ, Toi A Pitfalls in the ultrasonographic diagnosis of gallbladder disease. Postgrad Med $\mathcal{F}$ 1987; 63: 525-32.

4 Ekberg $\mathrm{O}$, Weiber $\mathrm{S}$. The clinical importance of a thick walled, tender gallbladder without stones on walled, tender gallbladder without st

5 Saverymuttu SM, Grammatopoulos A, Meanock CI, et al. Gallbladder thickening (congestive cholecystopathy) in liver disease; a sign of portal hypertension. Br f Radiol 1990; 62 $922-5$.

\section{Summary points}

- the normal gallbladder wall thickness should be $3 \mathrm{~mm}$ or less on ultrasound examination

- in our two cases, and in one of the cases reported in the literature, the presence of gallbladder wall thickening was associated with severe systemic illness in patients with infectious mononucleosis

Box 2

mononucleosis may be due to an immunological reaction similar to that which occurs in the liver, or perhaps due to oedema of the wall secondary to lymphatic obstruction due to enlarged nodes at the porta hepatis. Incomplete gallbladder distension from reduced bile flow secondary to hepatic dysfunction may also contribute. ${ }^{7}$

Both patients were extremely ill. We suggest that when gallbladder wall thickening is seen with infectious mononucleosis, it is usually associated with severe systemic illness, warranting close monitoring.

6 Talarico MP, Rubens D. Gallbladder wall thickening in acute pyelonephritis. $\mathcal{F}$ Clin Ultrasound 1990; 18: 653-7.

7 Hammond DI, MacLean RS. Gallbladder wall thickening in an elderly woman with infectious mononucleosis. $\mathcal{F}$ Clin Ultrasound 1987; 15: 558-60.

8 Maruyama $\mathrm{K}$, Ushiku $\mathrm{H}$, Kondou Y. Gallbladder wall thickening in children with infectious mononucleosis. $₹$ Clin Ultrasound 1994; 22: 576-8.

\title{
Gallstone ileus - beware the silent second stone
}

\author{
JB Davies, PC Sedman, EA Benson
}

\section{Summary \\ Gallstone ileus remains a rare but impor- tant cause of small bowel obstruction. We report a case of recurrent gallstone ileus, presumably caused by an unidentified second stone resident within the gallblad- der at the time of the initial laparotomy. This raises important questions about the traditional surgical management of this interesting condition.}

Diseases, The General Infirmary of Leeds, Great George Street, Leeds LS1 3EX, UK JB Davies PC Sedman

EA Benson
Keywords: gallstone ileus, recurrent stones

The incidence of gallstone ileus increases with age. In elderly patients it is often misdiagnosed and surgical treatment is associated with significant morbidity and mortality. Rarer still is the condition of recurrent gallstone ileus. This report highlights the various treatment options available to the surgeon.

\section{Case report}

A 63-year-old woman presented with a four week history of intermittent abdominal pain, vomiting and diarrhoea. Plain abdominal radiographs demonstrated proximal small bowel obstruction with air in the biliary tree. The clinical diagnosis of gallstone ileus was confirmed at laparotomy where a $4.5 \mathrm{~cm}$ nonfaceted gallstone (figure) was found impacted 